\title{
PI3Kalpha Inhibitor AZD8835
}

National Cancer Institute

\section{Source}

National Cancer Institute. PI3Kalpha Inhibitor AZD8835. NCI Thesaurus. Code C118624.

An orally bioavailable inhibitor of the class I phosphatidylinositol-4,5-bisphosphate 3kinase (PI3K) catalytic subunit alpha (PIK3CA), with potential antineoplastic activity. PI3K alpha inhibitor AZD8835 selectively binds to and inhibits PIK3CA and its mutated forms, in the PI3K/Akt (protein kinase B) /mammalian target of rapamycin (mTOR) pathway. This results in both apoptosis and growth inhibition in PIK3CA-expressing tumor cells. By specifically targeting PIK3CA, this agent may be more efficacious and less toxic than pan$\mathrm{PI3K}$ inhibitors. Dysregulation of the PI3K/Akt/mT OR pathway is often found in solid tumors and results in the promotion of tumor cell growth, survival, and resistance to chemo- and radio-therapy. PIK3CA, one of the most frequently mutated oncogenes, encodes the p110-alpha catalytic subunit of the class I PI3K. 